\title{
SPINDLE CELL MELANOMA HARBORING A NODULE OF EPITHELOID CELL MELANOMA COMPONENT: A STUDY OF A DIAGNOSTICALLY CHALLENGING CASE
}

\author{
Bartos V, Farkasova A. \\ Martin`s Biopsy Center, Ltd., Martin, Slovakia
}

\begin{abstract}
A b s t r a c t
Background: Melanoma is a very heterogeneous human neoplasm. In addition to four major (conventional) histologic subtypes a number of uncommon variants do exist.

Objective: An unusual case of a spindle cell melanoma (SCM) containing a demarcated nodule of conventional epitheliod cell melanoma component is described.

Material and Methods: A 71-year-old man manifested with a protuberated ulcerated skin tumor arising on the right forearm. The resected biopsy was analyzed immunohistochemically with a variety of anti-human antibodies.

Results: The tumor consisted of a highly cellular mass of spindle-shaped cells without any significant intratumoral fibrosis. In addition, a nodule of epithelioid cell tumor component was present within the lesion. The spindle cell component showed a disperse reactivity for S100 protein and was negative for other melanocytic markers. It exhibited a very high mitotic activity and proliferation Ki-67 index. No melanin pigment was detected. In contrast, the epithelioid cell component was strongly positive for S100 protein, Melan-A/MART-1, HMB-45, and PNL-2. The mitotic and proliferation indices were much less pronounced and melanin deposits were visible. A diagnosis of a non-desmoplastic SCM harboring a nodule of epithelioid cell melanoma component was established.

Conclusion: SCM often posses a diagnostic dilemma because its histomorphology is atypical and its immunohistochemical profile may differ from other subtypes of melanomas. The present paper points out this uncommon histopathological entity that may sometimes be encountered in dermatopathological practice and that requires more complex diagnostic approach.
\end{abstract}

Keywords: malignant melanoma, spindle cell component, epithelioid cell component, S100 protein

\section{INTRODUCTION}

Melanoma is one of the most aggressive malignancies in humans with a high mortality rate (1-3). Although it accounts for only $5 \%$ of all skin cancers, it is responsible for more than $77 \%$ of skin cancer-related deaths (2). This prognostically unfavorable phenomenon is exacerbated by the fact that the tumor has shown a rapid increase in incidence over the last few decades $(2,4)$. There are four major (conventional) histologic subtypes of cutaneous melanoma: superficial spreading melanoma, nodular melanoma, lentigo maligna/lentigo maligna melanoma, and acral lentiginous melanoma $(1,5)$. Although this classification has been accepted by many over the years, its artificiality and limitations have clearly been documented (5). In fact, melanoma is one of the most heterogeneous and complex human neoplasms (5). Consequently, on a regular basis one encounters melanomas that are difficult to categorize as one of the four major subtypes (5). A number of uncommon and unusual histologic variants have been described until now (Table 1) (5-7). Separating them from conventional subtypes mainly serves to remind pathologists of the morphologic diver-

Corresponding author: MUDr., PhDr. Vladimír Bartoš, PhD., MPH; e-mail: vladim.bartos@gmail.com (C) 2020 Bartos V. et al.

This work is licensed under the Creative Commons Attribution-NonCommercial-NoDerivs 4.0 License (https://creativecommons.org/licenses/by-nc-nd/4.0/) 
sity of melanomas and to point out diagnostic pitfalls (6). The recognition and delineation of both conventional and less common variants of melanoma correspond to a wide range of clinical, microscopic, genetic, and molecular phenotypic characteristics (5).

Table 1 Classification scheme of histological subtypes and variants of malignant melanoma. (5-7)

\section{A. Major (conventional) histologic subtypes}

1. superficial spreading melanoma

2. nodular nelanoma

3. lentigo maligna/lentigo maligna melanoma

4. acral lentiginous melanoma

\section{B. Uncommon histological subtypes and variants}

spindle cell melanoma

desmoplastic melanoma

neurotropic melanoma

nevoid melanoma

minimal-deviation melanoma

equine/animal-type melanoma

malignant blue nevus metaplastic melanoma

baloon cell melanoma

signet-ring cell melanoma

myxoid melanoma

small cell melanoma

rhabdoid melanoma

angiotropic melanoma

Melanomas with predominant spindle cell morphology comprises a spectrum of tumors. If associated with a marked fibrous stroma throughout the invasive component, they are designated desmoplastic melanoma (6). If intraneural and/or perineural invasion is prominent and a neuroma-like growth pattern is present, the term neurotropic melanoma may be applied (6). If the tumor does not have any of these features, it is called spindle cell melanoma (SCM), not otherwise specified (or non-desmoplastic SCM) (6). The main diagnostic problem related to melanomas with predominant spindle cells is their potential confusion with various types of sarcoma or sarcomatoid carcinoma (6). In this journal an interesting case of a desmoplastic melanoma has been recently published by Adamicova et al. (8). Herein, another unusual case of a non-desmoplastic SCM containing a demarcated nodule of conventional epitheliod cell melanoma component is described.

\section{CLINICAL SYNOPSIS}

A 71-year-old man manifested with a painless slow-growing tumor of the skin arising on the right forearm. On physical examination it appeared as a well-defined protuberated lightbrownish tumor mass. A presumptive clinical diagnosis was a hemangioma. A total surgical extirpation of the lesion was carried out at the Surgical Outpatient Department.

\section{MATERIAL AND METHODS}

The formalin-fixed resected biopsy consisted of the skin and subcutis $(25 \times 20 \mathrm{~mm})$ with a prominent dome-shaped ulcerated tumor measuring $17 \times 14 \mathrm{~mm}$. The specimen was processed into six paraffin-embedded tissue blocks which were stained with hematoxylin-eosin $(\mathrm{H} \& \mathrm{E})$. Selected sections were then analyzed immunohistochemically with a variety of antihuman antibodies (see below). 


\section{PATHOLOGY RESULTS}

Histology revealed a protuberated tumor with an extensive ulceration and a necrotic detritus at the base of the ulcer. It consisted of a highly cellular mass of atypical, gently eosinophilic spindle-shaped cells in a fascicular growth pattern without any significant intratumoral fibrosis (Figure 1). They showed vesicular cigar-shaped nuclei and numerous mitotic figures. In addition, a circumscribed nodule of epithelioid cell tumor component was visible within the lesion (Figure 2). This cell population had abundant, slightly basophilic cytoplasm with large round nuclei and prominent nucleoli and contained much less mitotic figures. At first glance, the two components were histologically clearly different, albeit both were undoubtedly malignant. A further analysis confirmed not only distinct histomorphology but also differences in the immunophenotype, mitotic and proliferation rates, and a presence of melanin. They are described in detail in the Table 2 . Briefly, the spindle cell component, which comprised the vast majority of tumor tissue, showed a disperse reactivity for S100 protein (Figure 3) and was negative for other melanocytic markers, i.e. Melan-A/MART-1 (Figure 4), HMB-45 (Figure 5) and PNL-2. It exhibited a very high mitotic activity and proliferation Ki-67 index (Figure 6). No melanin pigment was detected (Fontana-Masson staining). In contrast, the epithelioid cell component was strongly positive for S100 protein, Melan-A/MART-1, HMB-45, and PNL-2. The mitotic and proliferation indices were much less pronounced and melanin deposits were visible. Both tumor parts were positive for vimentin and negative for polyclonal cytokeratins (AE1/AE3), high molecular weight cytokeratins (HMWCK), CK5/6, p63, desmin, $\alpha$-smooth muscle actin, and CD34. In the contex of overall histopathology and immunoprofile, a final diagnosis of a non-desmoplastic SCM harboring a nodule of conventional epithelioid cell melanoma component was established. Other prognostic parameters (not listed in the Table 2) were as follows: Breslow index $6.9 \mathrm{~mm}$, vertical growth phase, Clark's level V, tumor-infiltrating lymphocytes and regression absent, lymfovascular invasion absent, perineural spreading present, and tumor microsatellite present. DNA isolated from the formalin-fixed and paraffin-embedded tissue (FFPE) material using Cobas ${ }^{\circledR}$ DNA Sample Preparation Kit was tested for the presence of the most common somatic mutations V600E, V600D, V600E2, and V600K of the BRAF gene. DNA was isolated from both histological components of the tumor separately. The testing was carried out at $\operatorname{cobas}{ }^{\circledR}$ Z480 platform (Roche Molecular Systems, Inc., CA, USA) using CE-IVD cobas ${ }^{\circledR} 4800$ BRAF V600 Mutation Test Kit (Roche Molecular Systems, Inc., CA, USA) following the manufacturer's instructions. These mutations can be detected with

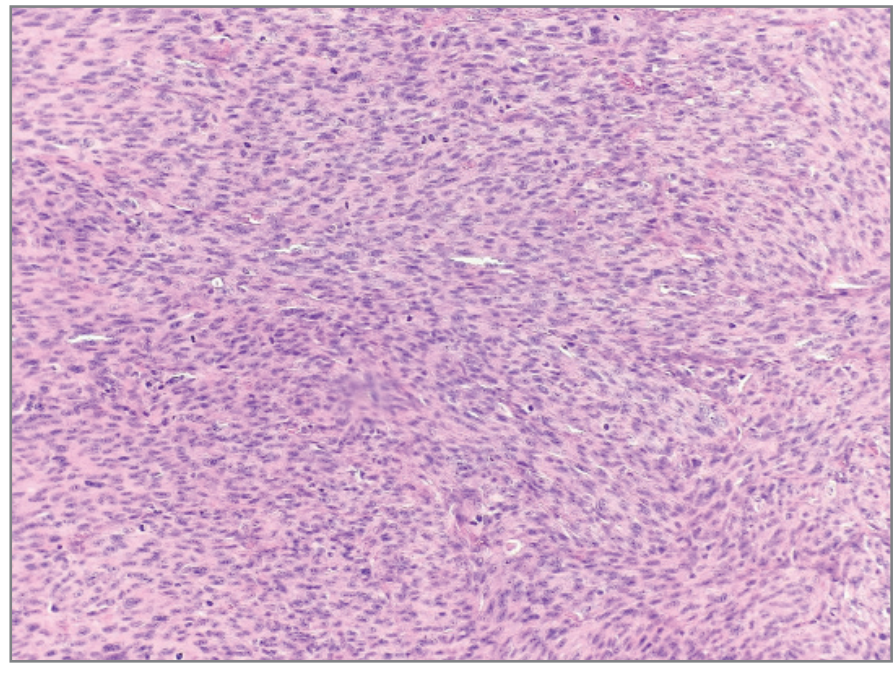

Fig. 1 Highly cellular mass of mitotically active spindle-shaped neoplastic cells. (H\&E, magnification 40x) 
a sensitivity of $\geq 5 \%$ mutant sequences in the background of wild-type DNA. The evaluation of the presence of the mutation was automatic using cobas ${ }^{\circledR} 4800$ version 2.1 software following the manufacturer's instructions. A molecular testing for BRAF gene mutations showed negative results in both tumor components.

After sending the final result of the pathologist's report, the authors have not had any information about further clinical management of the patient, but this matter was not a goal of this paper.

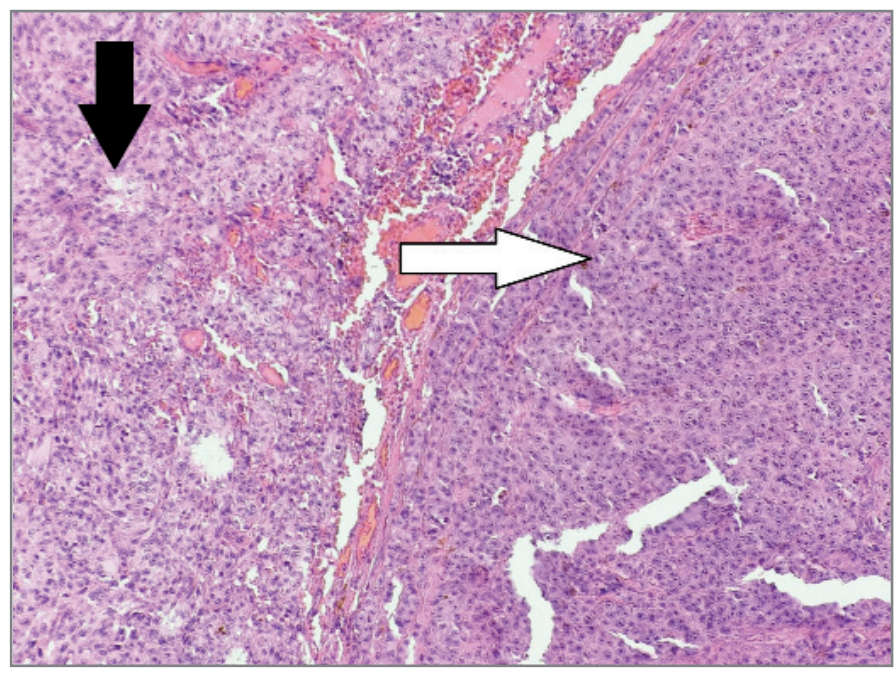

Fig. 2 Interface between a spindle cell component (left black arrow) and a nodule composed of epithelioid cell population (right white arrow). (H\&E, magnification 40x)

Table 2 Summary of selected findings in both tumor components. (mf/ $1 \mathrm{~mm}^{2}-$ number of mitotic figures per $1 \mathrm{~mm}^{2},{ }^{*}$ both typical and atypical mitoses were calculated)

\begin{tabular}{|l|l|l|}
\hline Parameter & Spindle cell component & Epithelioid cell component \\
\hline proportion of tumor mass & $90 \%$ & $10 \%$ \\
\hline mitotic rate & $>25 \mathrm{mf}^{*} / 1 \mathrm{~mm}^{2}$ & $7 \mathrm{mf}^{*} / 1 \mathrm{~mm}^{2}$ \\
\hline Ki-67 index & $90 \%$ & $25 \%$ \\
\hline ulceration & present & absent \\
\hline melanin pigment & absent & present \\
\hline necrosis & absent & absent \\
\hline S100 protein & disperse expression & diffuse expression \\
\hline melan-A, HMB-45, PNL-2 & negative & diffuse expression \\
\hline BRAF mutation & negative & negative \\
\hline
\end{tabular}



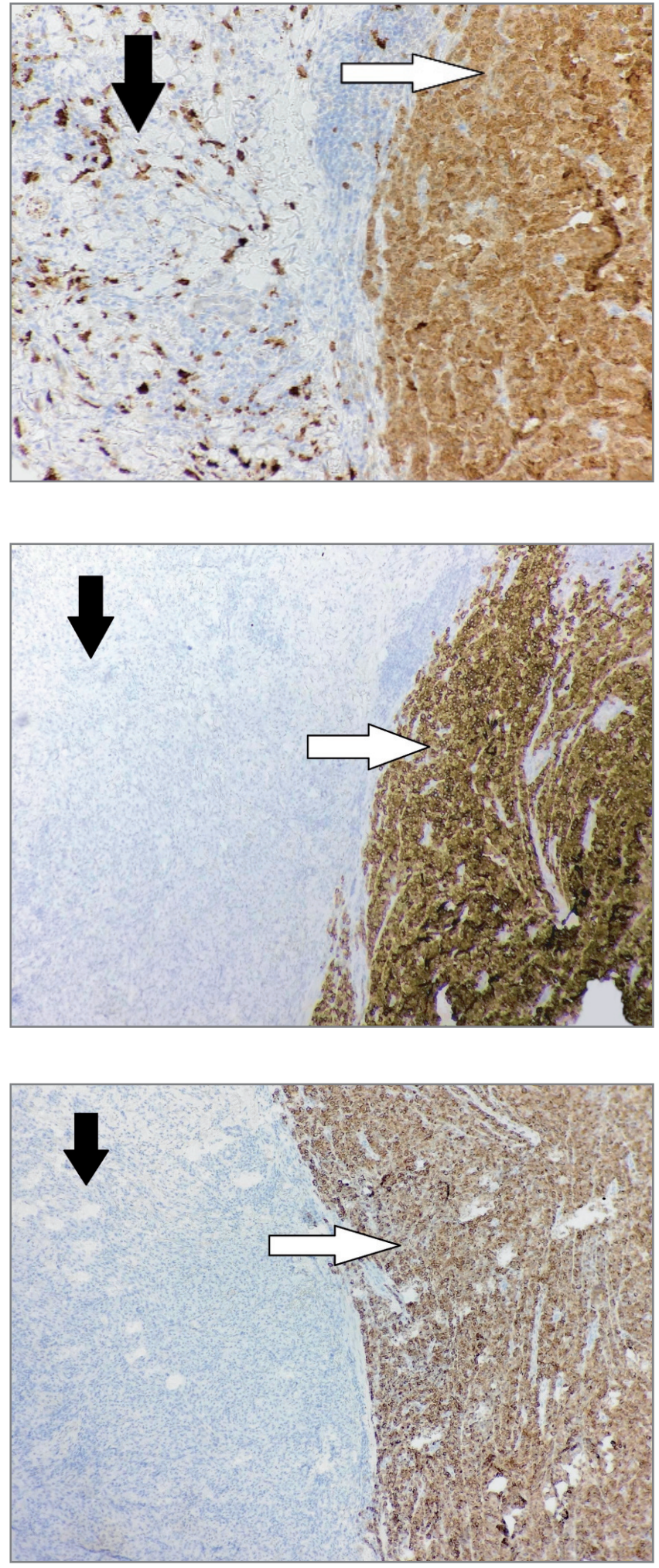

Fig. 3 S100 protein expression. Diffuse strong positivity in epithelioid cell component (right white arrow) and disperse positivity in spindle cell component (left black arrow). (magnification 40x)

Fig. 4 Melan-A/MART-1 expression. Diffuse strong positivity in epithelioid cell component (right white arrow), while spindle cell component is completely negative (left black arrow). (magnification 20x)

Fig. 5 HMB-45 expression. Diffuse strong positivity in epithelioid cell component (right white arrow), while spindle cell component is completely negative (left black arrow). (magnification 20x) 


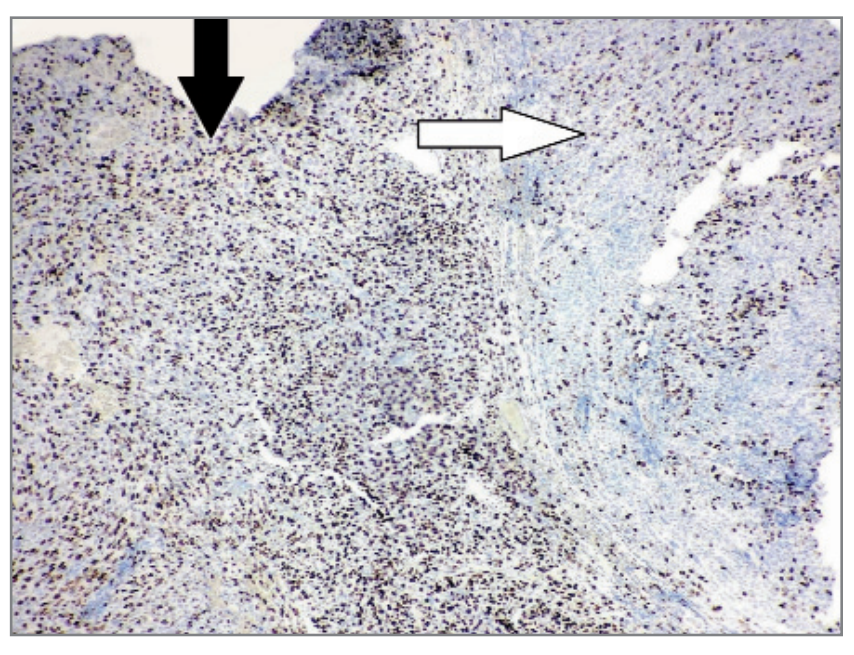

Fig. 6 Ki-67 antigen expression. Proliferation index is much more pronounced in spindle cell component (left black arrow) than in epithelioid cell component (right white arrow). (magnification 20x)

\section{DISCUSSION}

Spindle cell melanoma is an uncommon histologic variant of malignant melanoma composed of spindled neoplastic melanocytes arranged in sheets and fascicles $(6,9,10)$. In routine biopsy practice this term serves as an umbrella term for non-desmoplastic spindle cell and desmoplastic melanoma category. Its incidence has been variably reported between $3-14 \%$ of all melanoma cases (including desmoplastic melanoma) (9). However, an accurate occurrence is difficult to estimate. In a study of Minarikova et al. (4) no SCM variant has been mentioned among 112 new cases of malignant melanoma registered at the Skin Cancer Department at the Martin University Hospital in year 2017. The results may depend on the age distribution, sex, and ethnicity of the given study cohort. Non-desmoplastic SCM occurs more frequently in men with a median age of 66-68 years $(9,10)$, agreeing with our observation. In contrast to desmoplastic melanoma, which arises more frequently on the head and neck region $(1,8,10)$, non-desmoplastic SCM can occur essentially anywhere on the body, most often on the trunk (10). The diagnosing of SCM is usually challenging and awareness of its clinical and histopathological features as well as immunohistochemical markers are essential to reach the correct diagnosis (9). As a distinct nosologic entity, the SCM brings several diagnostic pitfalls. First, since it usually lacks melanin pigment it grossly mimics other amelanotic lesions $(6,9)$. In fact, amelanotic melanomas are great masqueraders that can mimic a range of skin pathologies including inflammatory lesions and also benign tumors such as hemangioma, pyogenic granuloma, lichen planus-like keratosis, or intradermal nevus that are not routinely excised (11). That is probably why the present case was also clinically considered a benign tumor, i.e. hemangioma. Second, the histopathologic features of SCM are often confused with those of other mesenchymal or epithelial neoplasms (9). Therefore, because of their atypical histomorphology, spindle cell and desmoplastic melanomas may reveal their identity as melanoma only after a first set of adjunct tests, typically including immunophenotyping (10). Third, although an immunohistochemistry is a crucial tool in distinguishing SCM from other malignancies, the SCM may show an unusual immunophenotype and even be negative for classic melanocytic markers (10). Those that are more specific for conventional melanoma generally show poor sensitivity for SCM lesions. American investigators (12) reviewed a series of papers that studied markers for their capacity to detect spindle cell and desmoplastic melanomas. Among them, the S100 protein showed a sensitivity of $98.7 \%$, but the rates of positive staining for Melan-A/MART-1 and HMB-45 were only $21.6 \%$ and $17.6 \%$, respectively (12). Newer antibody PNL-2 have not 
yielded more promising results. In a study of Busam et al. (13) only 1 of 13 desmoplastic melanomas (7.7\%) reacted with PNL-2. In this regard, the S100 protein is by far the most reliable marker. The present case is excelent for demonstrating the different immunophenotypes of two distinct melanoma variants within a single lesion. Such cases are quite uncommon in dermatopathologic practice. Particularly the presence of a conventional nodular epithelioid melanoma component exhibiting a typical positivity for all melanocytic markers was very helpful for establishing the final diagnosis. The coexistence of two histologic variants of melanoma within a single lesion is a consequence of intratumor phenotypic heterogeneity. It has been well documented $(1,14,15)$ that the mutation rate of melanoma is very high and it strikingly increases with tumor progression. A high number of clones harboring various mutations contribute to an exceptional level of intratumor diversity in melanoma. Even metastases may originate from different subclones of the primary lesion (14). In our case there were undoubtedly at least two different neoplastic cell clones. Further research addressing this issue is very perspective since levels of tumor heterogeneity in advanced-stage melanomas hinder accurate diagnosis and effective treatment and adversely affect a personalized cancer medicine strategy. Currently, the only biomarker that predicts a therapeutic response to so called target therapy in advanced melanoma is a BRAF gene mutation status (15). Targeted therapy with BRAF inhibitors is associated with significant long-term treatment benefit in patients with BRAF-mutated melanoma (15). Therefore, molecular testing for BRAF mutations is a priority in determining the course of therapy. Although the BRAF V600 mutations have been detected only in $31 \%$ of spindle cell and $5 \%$ of desmoplastic melanomas (10), given the treatment implications, it argues for standard BRAF testing even in such uncommon melanoma subtypes.

\section{CONCLUSION}

Spindle cell melanoma often posses a diagnostic dilemma because its histomorphology is atypical and its immunohistochemical profile may differ from other subtypes of melanomas. The present paper points out this uncommon histopathological entity that may sometimes be encountered in dermatopathological practice and that requires more complex diagnostic approach.

\section{REFERENCES}

1. Elder DE, Massi D, Scolyer RA, Willemze R. WHO Clasification of Skin Tumours. 4th Edition, IARC Lyon, 2018. p. 66-152.

2. Calkovsky V, Minarikova E, Bobrovska M, Hajtman A. Malignant melanoma of the neck. Acta Medica Martiniana 2008;8(3):37-44.

3. Kozarova A, Minarikova E, Pappova T. Practical use of high-resolution $20 \mathrm{MHZ}$ ultrasonography in dermatovenerology. Acta Medica Martiniana 2016;16(3):22-31.

4. Minarikova E, Smolarova M, Minarik M. Activity of skin cancer clinic at Martin University Hospital in 2017. Acta Medica Martiniana 2018;18(1):21-29.

5. Barnhill RL, Sarantopoulos GP, Gupta K. Less common variants of cutaneous melanoma. In: Sharfman WH, Abraham J. Melanoma (Emerging Cancer Therapeutics, Vol. 3, Issue 3), 1st Edition. Demos Medical, 2012, p. 421-460.

6. Busam KJ. Dermatopathology. A Volume in the Series: Foundations in Diagnostic Pathology. 1st Edition. Saunders Elsevier, 2010, p. 478.

7. Magro CM, Crowson AN, Mihm MC jr. Unusual variants of malignant melanoma. Modern Pathology 2006;19:S41-70

8. Adamicova K, Fetisovova Z, Bobrovska M, Homola I. The importance of using SOX10 for exact diagnosis of desmoplastic melanoma. Acta Medica Martiniana 2019;19(3):112-116. 
9. Xu Z, Shi P, Yibulayin F, et al. Spindle cell melanoma: Incidence and survival, 1973-2017. Oncol Lett 2018;16(4):5091-5099.

10. Weissinger SE, Keil P, Silvers DN, et al. A diagnostic algorithm to distinguish desmoplastic from spindle cell melanoma. Mod Pathol 2014;27(4):524-534

11. Smolarova M, Minarikova E. Dermoscopy as a valuable tool in diagnosis of nodular amelanotic melanoma and nodular basal cell carcinoma. Acta Medica Martiniana 2017;17(1):21-27.

12. Ohsie SJ, GP Sarantopoulos, Cochran AJ, Binder SW. Immunohistochemical characteristics of melanoma. J Cutan Pathol 2008;35(5):433-444.

13. Busam KJ, Kucukgol D, Sato E, et al. Immunohistochemical analysis of novel monoclonal antibody PNL2 and comparison with other melanocyten differentiation markers. Am J Surg Pathol 2005;29(3):400-406.

14. Grzywa TM, Pascal W, Wlodarski PK. Intratumor and intertumor heterogeneity in melanoma. Transl Oncol 2017;10(6):956-975.

15. Cheng L, Lopez-Beltran A, Massari F, et al. Molecular testing for BRAF mutations to inform melanoma treatment decisions: a move toward precision medicine. Mod Pathol 2018; 31(1):24-38.

Received: January 18, 2021

Accepted: February, 8, 2021 